\title{
EXIGENCIAS EPISTEMOLÓGICAS: EL RECORRIDO DE CARMELO LISÓN TOLOSANA
}

\author{
Epistemological Requirements: The journey of Carmelo \\ Lisón Tolosana
}

\section{Requisitos epistemológicos: A trajetória de Carmelo Lisón Tolosana}

\author{
Bernard TRAIMOND \\ Universidad de Burdeos \\ bernard.traimond@u-bordeaux.fr \\ Fecha de recepción: 12/01/2021 \\ Fecha de aceptación: 8/02/2021
}

RESUMEN: El autor indaga en los aspectos innovadores de la obra de Carmelo Lisón Tolosana, formado en el escuela estructural-funcionalista británica, pero con una perspectiva historicista, que engarza con ciertas antropología francesas, aunque no tengamos constancia de directas influencias mutuas. Entre otros puntos en común entre esas antropologías y las de CLT, tenemos, las "escalas microscópicas", el punto de vista del hablante, la atención a las palabras y las formas de expresión, la contextualidad de las informaciones, y en definitiva el proceso hermenéutico de conocimiento.

Palabras clave: hermenéutica; escala; emic/etic; contexto; Lisón Tosana; Evans-Pritchard.

ABSTRACT: The author investigates the innovative aspects of the work of Carmelo Lisón Tolosana, trained in the British structural-functionalist school, but with a historicist perspective, which connects with certain French anthropology, although we have no evidence of direct mutual influences. Among other points in 
common between these anthropologies and those of CLT, we have, the "microscopic scales", the speaker's point of view, attention to words and forms of expression, the contextuality of the information, and ultimately the process hermeneutic of knowledge.

Key words: hermeneutics; scale; emic/etic; context; Lisón Tosana; Evans-Pritchard.

RESUMO: O autor indaga nos aspectos inovadores da obra de Carmelo Lisón Tolosana, formado na escola estrutural-funcionalista britânica, porém com uma perspectiva histórica, que se vincula com certas antropologias francesas, ainda que não tenhamos constância de diretas influências mutuas. Entre outros pontos em comum destas antropologias e as de CLT, temos, as escalas microscópicas", o ponto de vista do falante, a atenção e as palavras e as formas de expressão, a contextualidade das informações e em definitivo o processo hermenêutico de conhecimento.

Palavras-chave: hermenêutica; escala; emic/etic; contexto; Lisón Tosana; Evans-Pritchard.

En 1974, Carmelo Lisón Tolosana publica en la Revista Española de Opinión Pública un artículo titulado «Sobre la Antropología cognitiva: el arresponsador gallego». El azar me hizo leerlo inmediatamente en el volumen Perfiles Simbólicos y Morales de la Cultura Gallega, publicado el mismo año en la editorial Akal. Recuerdo haberle preguntado al profesor de español del colegio donde yo trabajaba, el sentido de la palabra "arresponsador". Me dijo: "responso", "palabras, ordinariamente sacadas de las Escrituras que se dicen o se cantan, en los oficios de la Iglesia», nos informa el Dictionnaire de Littré. Después me he acercado a la vía más o menos abierta por este artículo. Es decir, el lugar de Lisón en su propia historia intelectual. Hablaré, pues, a la vez de la vía de Lisón pero también de aquellos que marchan tras sus pasos.

De principio voy a volver una vez más sobre su obra, ya que él no está aquí para que yo pudiera interrogarlo sobre tal o cual punto oscuro que corregiría mis consideraciones. Puedo desgraciadamente, en consecuencia, escribir sin arriesgarme a ser desmentido pero también sin su aprobación o su crítica ${ }^{1}$. El artículo fundacional invocado más arriba va a permitirse demostrar tres cosas. De un lado, ¿cómo Carmelo ha hecho para salir del folclorismo reinante en la antropología europea? De esta manera, consecuentemente, propondría los paradigmas de esta disciplina para el medio siglo que seguiría. En fin, en tercer lugar, yo presentaré

1. Cuando le he enviado la noticia sobre él realizada para Berose, Encyclopédie internationale des histoires de l'Anthropologie, Carmelo me ha respondido el 5 de febrero de 2019: "Nadie conoce mejor que tú, mi obra». Era evidentemente falso, pero lo que él me notificaba es que el artículo le gustaba, aunque me había señalado algunos detalles que debía añadir. 
los trabajos de sus descendientes que probablemente no conocen su obra, pero que trabajan en la perspectiva que él ha trazado, y que no puede más que imponerse hoy día.

\section{QUÉ ESTRATEGIA CONTRA LOS CONFORMISTAS}

Igualmente, aunque su vocación sea la investigación y en consecuencia la novedad, demasiado frecuentemente la Universidad se complace en el academicismo, en «la reproducción pomposa de los maestros conocidos» (Geertz, 1973). No solamente no innova generalmente, sino peor todavía, combate toda idea nueva. "El mal viento que viene de América», así denunciaba un antropólogo francés el soporte en el que Michel Foucault había llamado el «nacisismo monológico». Lo que significa que todo investigador un poco creativo se enfrenta con un muro con el cual debe componer de una manera u otra.

\section{II. "COMMUNITARY STUDIES"}

En este combate, miremos cómo ha procedido Carmelo Lison más o menos conscientemente. Ya he evocado esta cuestión en el artículo «La novedad de la obra de Carmelo Lisón Tolosana" (Traimond, 2012), a propósito de los "Communitary Studies». Retomando una antigua formulación que disponía también de un nombre, la corografía, a partir de 1930 las obras de los esposos Lyndt (Littletown) y Robert Redfield (Tepozland) reintrodujeron este antiguo objeto surgido de la erudición local en el mundo académico. Después, unos cientos de investigaciones han tomado como sujeto una comunidad como vía de acumulación de informaciones heteróclitas pero localizadas. Puede ser que fuese una reacción contra las generalidades del formalismo en boga en la misma época. Sin un recuento sistemático, he podido registrar 300. La mayor parte, clasifican las informaciones recogidas en grandes conjuntos supuestamente vinculados, economía, política, religión... En su tesis, Lisón retoma el camino señalado en su título Belmonte de los Caballeros, pero la lectura propone otra perspectiva. Si él se sitúa en una comunidad, espacio restringido reclamado por toda investigación antropológica, propone una cuestión: ¿qué valores allá organizan discursos y conductas? Su investigación quiere responder al problema propuesto, pero sobre todo elude el eclecticismo, las normas implícitas (la dominación de la economía) y sobre todo acopió informaciones heteróclitas. De esta constatación hecha a propósito de Belmonte de los Caballeros, concluyo a propósito de la postura de Lisón: "Tomar la doxa del momento, presentar sus apariencias para abrir nuevas vías, tal me parece su programa de trabajo, sea o no plenamente consciente de ello» (Traimond, 2012: 57). 


\section{ESTRUCTURALISMO}

Cuando hace sus primeros escritos alrededor de 1970, el «espíritu de la época", al cual necesariamente se confronta Carmelo Lisón, surge del formalismo estructuralista. Por entonces participa en unas "Conferencias y seminarios en torno al tema estructuralismo", organizadas por la Facultad de Filosofía y Letras de la Universidad de Sevilla. La intervención, "Estructuralismo y antropología", publicada el mismo año en la Revista Española de Opinión Publica, fue retomada en 1971 en su primer libro Antropología social en España, que el azar me hizo comprar desde su aparición.

Esta vez, sin investigación de archivos u otros informantes para comenzar la demostración como Lisón hace a lo largo de toda su obra, puede ser la única en la que se apoya en el razonamiento proponiendo unas nociones para identificar las contradicciones, ilustradas en unos ejemplos imaginados por la circunstancia, en la perspectiva de los filósofos, de su "régimen de verdad» y su poética. Así, sitúa en el terreno de esta última, no en el de la antropología, todo como el proyecto estructuralista que se otorga como fin, el viejo asunto del conocimiento del espíritu humano.

Al final de su artículo, Lisón se opone, por tanto, a la idea de que «las relaciones entre las partes determinan el estilo, la forma, la estructura, en una palabra»: inmediatamente, privilegia la parte sobre la totalidad. Pero para desmontar esta idea -fundamento del estructuralismo- propone unas categorías que separan dos binarismos, la "estructura concreta» / analítica» y la «estructura real»/«ideal». Esto es propuesto en dos páginas, Carmelo pasa inmediatamente a otro registro, «el aspecto positivo y práctico» del sistema de herencia de las tierras agrícolas en Galicia, al cual yo accedo de otro lado fácilmente ya que es el mismo que existe entre nosotros, en las Landes de Gascuña: para mantener la viabilidad de la explotación, el primogénito hereda los 2/3 de las propiedades: los menores se repartirán el resto. Lisón puede entonces presentar las tensiones y los conflictos nacidos de estas situaciones tanto como los viejos conservan sus bienes y su autoridad hasta su muerte. Llama a estas relaciones nacidas de estas situaciones, «estructuras» y para mostrarlo, reemplaza las palabras por los esquemas, las interacciones por las flechas. La reducción de las palabras y de las situaciones es drástica. ¿Por qué no? Pero a condición de precisar los peligros y los límites. Si bien reencontramos así los esquemas que tanto amaba Lévi-Strauss, somos conducidos más lejos de lo que es habitualmente llamado «estructuras» y aún más de las concepciones del antropólogo francés.

Aquí entonces, Lisón emplea el procedimiento habitual que yo había definido mientras vivía sin que se indignara, subvertir un aparente conformismo. Cuando he avanzado esta idea hace diez años, no pensaba poder verificar tan fácilmente su pertinencia a propósito de sus relaciones con el estructuralismo. 
Pero es peor, cuando le había hablado en marzo de 2019 me parece, de lo que le había preguntado lo había olvidado ${ }^{2}$. Al final de su texto sobre el estructuralismo, Lisón emplea la expresión "situación crítica». O esta última no es inocente porque proviene de Karl Jasper, retomado en Francia por Gabriel Marcel y en fin utilizada por par Jean-Paul Sartre en La Nausée (1938). Así, para hablar de estructuralismo, Lisón emplea subrepticiamente las categorías de sus críticos (Traimond, 2004: 83), no solamente hablando de "situación", sino retomando algunos "conceptos» importantes de este "existencialismo» que rechazaba tanto a Lévi-Strauss.

\section{EMIC/ETIC}

Por consiguiente, para no romper con la comunidad antropológica, Lisón ha escogido retomar el binarismo etic/emic ${ }^{3}$, uso que me estorbaba en lo que consideraba como su obra maestra y que yo he logrado hacer traducir al francés, La Santa Compaña. Pero miremos más detenidamente cómo presenta el asunto (Lisón, 1998: 169): "El juego narrativo -local, emic- con frontera no solo integra sucesos y contexto o conjunta enumeración y referencia para producir efecto de lo real como acabo de sugerir, muestra también la facies cultural que armoniza la politópica yuxtaposición de opuestos e incompatibles. Pero el cronotopo frontera es, además y principalmente, una categoría antropológica, una figura etic por medio de la cual intentamos entrar contexto o conjunta enunciación y referencia para producir el contacto con un inaccesible mundo espiritual, misterioso y fantástico». Estas dos frases presentan toda la vía de Lisón que a mí me parece particularmente nueva y fecunda. Es por lo que yo amo este libro. Juzguémoslo:

Lo "local, emic" se expresa por una narración en la cual aparecen todos los elementos conocidos de la realidad, los acontecimientos, el contexto, los procedimientos retóricos y la "cultura", tanto elementos empíricos y, por consiguiente, heteróclitos. Sin embargo, desde el exterior se puede imaginar un mundo «espiritual, misterioso fantástico». Consecuencia, ¿de qué debe ocuparse la antropología? ¿Sus investigaciones presentan una realidad verbalizada caótica o un relato armonioso? Escoger el primer aspecto no supone para nuestros hablantes concebir su «mundo inaccesible» ${ }^{4}$.

2. En revancha, y esto confirma mi propósito, cuando yo había evocado delante de él la redacción de una obra sobre la influencia de Sartre en la antropología, adujo sus relaciones con el filósofo francés.

3. No es menos interesante anotar que yo he descubierto este binarismo en España y luego en Bateson. No sólo el binarismo se encuentra en el Dictionnaire de l'ethnologie de Michel Panoff y Michel Perrin (1973) sino en 1998, cuando era posible publicar en L'Homme, la revista creada por Lévi-Strauss, un artículo sobre la cuestión: J. J. Olivier de Sardan, «Emique».

4. La interpretación que hago del empleo de emic/etic en la recensión que hice en 2012 de la Santa Compaña para la revista Ethnologie Française - asimismo si el autor la mantiene, no será por 
Por mi parte, resolví la dificultad reduciendo tarea del investigador al trabajo sobre "lo emic" tal como lo verbalizan los actores y testigos que dan unas informaciones sobre las que puede establecer el crédito para la "crítica histórica». Sin embargo, más allá, el lector y el hablante pueden legítimamente imaginar unos objetos abstractos verificables que tienen perfectamente el derecho de imaginar por diferentes razones, apuestas morales o políticas en particular. Como el investigador no puede probar este tipo de especulaciones, debe pararse antes, no presentar lo que es posible afirmar sin contestación posible. Dejemos la «historia narración" al lector y a la ficción para dirigirnos a la "historia investigación" según la distinción de Aristóteles como la presenta Jablonka (2017: 146). Lisón llama esta distinción etic/emic para consagrarse al segundo término.

\section{LOS PARADIGMAS}

Mostrando lo que Lisón rechazaba, he comenzado a desvelar lo que reclamaba. Puedo entonces ahora de manera un poco dogmática presentar los paradigmas sobre los cuales se apoya para la descripción de los principios ya perfectamente presentes en el artículo en mi opinión en «Sobre la Antropología cognitiva: el arresponsador gallego» del cual he hablado.

\section{V.1. La escala}

La escala "microscópica» en este sentido que el investigador no puede encontrar más que en algunos hablantes que representan a nadie más que a ellos mismos. Pero esto permite establecer una complicidad con el investigador con dos secuencias. La primera es que el hablante verbaliza sus prácticas lo que no habría hecho nunca sin la intervención del antropólogo. La segunda es que la complicidad compartida lo autoriza expresar unas cosas que no habría confiado nunca a nadie más. El antropólogo accede de esta manera a unas vistas estrechas pero precisas, delimitadas pero netas, limitadas pero aseguradas. Dispone entonces, tras el examen crítico, de las mejores informaciones posibles.

\section{V.2. El objetivo, el acceso al punto de vista del hablante}

Sobre todo, este diálogo con los actores y los testimonios permite acceder a su punto de vista, al lugar desde el que ellos hablan, al contexto de sus prácticas, a los objetivos que se dan. Las informaciones son parciales pero reveladoras de las concepciones del hablante y de la manera en la cual él vive y formula sus propias

razones de presentación, las categorías de objetivo y subjetivo, de etic y de emic»- era falsa porque era sólo una concesión a lo habitual en los antropólogos para mejor subvertirlos. 
experiencias, su "conciencia verbal» para retomar la fórmula de Gérard Althabe (1969: 296). Evidentemente, se expresa bajo el imperio del «secreto, silencio y mentira", para retomar las palabras de Visweswaran (1994: 60) pero la crítica de las informaciones nos da los medios de jerarquizarlos desde los más absurdos hasta los más verosímiles.

\section{V.3. La atención a las palabras y a las formas de su expresión}

Pero tan sutiles como sea los análisis de las palabras pronunciadas, ellas dejan demasiado frecuentemente de lado la manera de expresarse. Por tanto, Malinowski, retomado por Jakobson, había inventado la "lengua enunciada", la cosa y la palabra, con el fin de designar todo lo que presenta la palabra fuera de su sentido literal. Más tarde, Alessandro Duranti ha forjado la palabra "etnopragmática» que analiza las palabras registradas en el curso de la investigación por medio de la pragmática del lenguaje, rama de la lingüística salida de Wittgenstein y Austin que se ocupa de las «relaciones entre lenguaje y contexto que son gramaticalizadas o incluidas en las estructuras del lenguaje», según la definición de Levinson (1983: 9). Los propósitos de los hablantes y las maneras de expresarlos constituyen pruebas lingüísticas de la realidad de las situaciones atravesadas. En el texto se encuentran las trazas del contexto.

\section{V.4. Contextualización de las informaciones}

Es decir que cada información nace de una experiencia y se formula en ciertas circunstancias, tanto de elementos que contribuyen a su nacimiento como también a su forma y a su comprensión. Ella no puede entonces ser empleada más que teniendo en cuenta las condiciones de su génesis y de su expresión, "enunciación y referencia» nos dice Lisón. Esta posición descalifica no solamente todas las formas cifradas -igualmente si Lisón se dejar tentar en las páginas 46-48 de La Santa Compaña- pero también toda reducción y abstracción.

\section{V.5. En fin, hacer del proceso de investigación un instrumento de conocimiento}

Como la investigación pasa necesariamente al lado de ciertas informaciones, como el investigador las orienta por su estatuto mismo, yo no puedo más que retomar las frases definitivas de Crapanzano. "Nuestras construcciones deben ser juzgadas en términos de relaciones -las posibles comprensiones e incomprensiones- que nacen de nuestra manera de abordar os otros. Ellas transitan por el lenguaje y nuestra percepción del lenguaje, por la traducción y nuestra comprensión de la traducción, por el relato y las convenciones descriptivas y nuestro reconocimiento crítico de las convenciones, por nuestras capacidades proyectivas 
y nuestra evaluación de estas capacidades. Ellas transitan también por la comprensión que tiene nuestro interlocutor de estos mismos factores» (Crapanzano, 2012:19). Desde entonces el único medio de tener en cuenta todas las distorsiones consiste en presentarlas al lector, de notificarle el detalle del desarrollo de la investigación, sus condiciones, sus dificultades, sus apuestas.

Encontramos las aleatoriedades de la investigación del antropólogo que a diferencia de los historiadores fabrica sus propios documentos. Esto significa que el investigador puede potencialmente encontrar siempre unas informaciones suplementarias, lo que lleva a Chauvier a decir que «la investigación está condenada a continuar» (Chauvier, 2006: 132). Yo había comparado el antropólogo a un viajero sentado en el sentido de la marcha de un tren que ve cada vez más detalles del objeto atisbado a lo lejos que ha escogido examinar. A la inversa, instalado en sentido inverso, el historiador no puede encontrar otras informaciones más que aquellas que de proporcionan los documentos accesibles. La comparación se impone tanto más cuanto que Lisón ha practicado las dos disciplinas. La fascinación de la diferencia (2005) al igual que Demonios y exorcismo en los siglos de oro (1990) son dos libros de historia. El segundo se apoya en los impresos consagrados al objeto indicado en el título. Lisón cita los textos de la época, así como sus comentarios posteriores. Pero necesariamente pocos testimonios de los actores ya que ellos forman lo esencial de las informaciones empleadas en La Santa Compaña (1998). De otro lado, si los interrogatorios de policía cualquiera sea su forma han autorizado tan bellos libros como Montaillou, village occitan de Le Roy Ladurie a Le fromage et les vers de Ginzburg. En ellos se atraviesan los siglos a través de las palabras de los actores. «Lo que falta quizás es la mirada directa: el testimonio, sin intermediario, que lleva el campesino sobre él mismo", escribe Le Roy Ladurie desde las primeras líneas de su Montaillou (Le Roy Ladurie, 1975: 9).

Podemos concebir sin pena las influencias que han llevado a Lisón a inscribirse en los paradigmas enunciados más arriba. Yo veo dos. La primera proviene de sus estudios de historia en la Universidad de Zaragoza donde necesariamente se ha iniciado en la crítica a las fuentes, pero también en la mirada del investigador que establece el crédito relativo a acordar al documento que ha consultado. Aquel que lo ha redactado, su lugar en el acontecimiento que relata (actor, testigo o comentarista), su historia personal y social... constituyen otros tantos elementos a tener en cuenta. En efecto, en los recursos reiterados en el examen de los objetos abstractos legitimado por las consideraciones puestas como evidentes tal como la objetividad, el grupo, la distancia, la teoría... la disciplina histórica mantiene el imperativo de emplear fuentes de primera mano, constatadas y no deducidas según la distinción de De Certeau. Transportado a la antropología, esta necesidad exige el recurso a las investigaciones, diálogo entre el investigador y sus informantes, exigencia que Lisón no ha cesado de repetir.

La segunda influencia ha surgido de sus estudios en Oxford con Evans-Pritchard del cual él ha tomado lo mejor, es decir el análisis de las situaciones magníficamente ilustrado por "la conversación a la orilla del río Nyanding» en Les Nuers 
(Evans-Pritchard, 1968: 7), la discusión entre Cuol y el antropólogo. En 2008, yo escribí que "este diálogo aparece como el blasón de la antropología bordelesa" (Traimond, 2008: 67).

\section{LOS HIJOS DE LISÓN}

Los paradigmas en los que se apoya Lisón no pueden ser adoptados más que los antropólogos más interesantes, aunque no lo hayan leído necesariamente. Un índice de esta continuidad, de esta "comunidad de lenguaje», fórmula bordolesa de la "Conversación a la orilla del río Nyanding" (Evans-Pritchard, 1968: 22) invocado sucesivamente por Bennetta Jules-Rosette (1996), Eric Chauvier (2004) y Bernard Traimond (2008).

Bennetta nos había situado bajo los ojos este diálogo: "A nivel semiótico el sujeto etnográfico y el etnógrafo cambian sus posiciones dialógicas. El objeto de valor para el etnógrafo -la filiación de Cuol- es abandonado en favor del equilibrio de la interacción. Es necesario anotar que las filiaciones entre los nuer representan lazos políticos y militares. En consecuencia, las filiaciones ocultan, de alguna manera, unos secretos militares. Acto seguido, es necesario constatar que este diálogo, ilumina un juego de poder y de dominación. El saber es, entonces, esta vez poder. En fin, este diálogo desvela un intercambio de dones, de anticipaciones y de informaciones. La conversación entre Evans-Pritchard y Cuol es un ejemplo de antropología dialógica fallida (Jules-Rosette, 1996: 74). Jules-Rosette presenta de manera reunida todos los recursos de algunas réplicas transcritas, que contextualizadas, presentan lo que dicen y,sobre todo, lo que ocultan.

Leamos las consecuencias que extrae Chauvier: "Evans-Pritchard se sirve aquí de la restitución de estos los entresijos de las encuestas para mostrar al lector que una experiencia científica entre los nuer es una cosa difícil, de la que el lector no duda pasando por la introducción. ¿Pero es este el único interés de este extracto? ¿El lector no se ha puesto delante de un cierto saber sobre la comunidad nuer, un saber de otra forma, que observa a largo plazo, y puede que abusivamente ser calificado - precisamente- de "saber entre bastidores"? Si este saber, que aparece al margen de la ciencia, sitúa mal el prestigio del antropólogo, no comporta menos un valor heurístico. En el ejemplo relatado, esto «sería, según las propias palabras de Evans-Prichard, dar a ver "la manera de sabotear una investigación». Detrás de este «sabotaje», este punto de detalle que parece externo a las preocupaciones del antropólogo, aparecen en efecto algunos aspectos fuertes de las prácticas lingüísticas de los nuer, hasta el punto que esto es dado por una falla, o al menos por una

5. Antropóloga, aunque profesora de sociología en la Universidad de San Diego en California, Bennetta Jules-Rosette ha dirigido de 1995 a 1999 el centro de California de Burdeos; participando activamente en los seminarios del Centre d'Etudes et de Recherches Ethnologique, ha conformado grandemente ciertas convicciones. Como Lisón, ella ha estado próxima a Víctor Turner y James Fernández. 
dificultad al investigar, a saber "la manera de sabotear una investigación», podría constituir una categoría clasificatoria en parte entera. No se trata evidentemente de descreditar las investigaciones más oficiales y más avanzadas de Evans-Pritchard, sino de considerar que las rupturas de comunicación, los sabotajes de la investigación, y para decirlo todo, la restitución de la posición natural (entendiendo por esto el antes de que las convenciones de análisis sean adoptadas) de EvansPritchard en su terreno, son reveladoras de un saber que presenta la ventaja de resistir a las categorías genéricas de la antropología. Este saber al margen, que sugiere antes de que se cierre (el extracto dialógico relacionado guía la imaginación del lector), permite al final delimitar las prácticas más conocidas de los nuer (Chauvier, 2004: 157-158).

Yo retorno sobre el dossier cuatro años más tarde (Traimond, 2008: 69): «Proponemos diferentes justificaciones al silencio de Cuol. En primer lugar, es inútil hablar a los imbéciles, estos europeos que vienen a nosotros (...) Acto seguido, nos dice Bennetta Jules-Rosette, el diálogo expresa unas relaciones de poder. El uno osa proponer preguntas sobre temas secretos, el otro está divertido practicando la evasión, para ganar tiempo, en cambiar de objeto y proponer otras cuestiones para no responder todo mientras continúa la conversación, el equilibrio de la interacción. En fin, el diálogo expresa unas estrategias de intercambio de bienes y de informaciones (...)».

He aquí unas glosas sobre treinta líneas solamente. Más allá de la masa de informaciones explícitas e implícitas que ellas proponen, de manera más general, muestran la riqueza de un simple diálogo, tan breve como sea. Una vez entonces, el análisis profundo de una situación permite acceder a unos conocimientos mayores. En antropología, «The bridge» de Max Gluckman (1940) o «Le combat de coqs» de Geertz pero más aún, en otra disciplina, Mimesis de Auerbach (2000), demuestran que el examen profundizado de una situación puntual permite acceder al conocimiento "serio" del conjuntos más extensos.

Empero el camino abierto por Lisón toma otros caminos como aquellos ya descritos (Traimond, 2012) cuando tengo presentes los trabajos de Eric Chauvier, Colette Milhé et François Zumbiehl tomados arbitrariamente entre otros muchos. Podría dar otros nombres como Anne Doquet, Julie Campagne, Yann Beldame, Eric Perera, Ariane Monnier... y más que se inscriben en la misma línea. Por tanto, con ellos ha iluminado un aspecto nuevo, la atención soportada por la escritura. Encarnada en Francia por Chauvier, esta preocupación se ha extendido desde hace poco a la historia que como la antropología «es una literatura contemporánea” para retomar el título del libro de Jablonka (2017). De esta manera, no solamente ciertos trabajos de Chauvier han sido leídos quizás como ficciones sino que esta última dimensión es reivindicada por Colette Milhé (2020): ella se atreve a presentarnos lo que su interlocutor debe pensar, aspecto habitualmente oculto en las investigaciones en que todas las preguntas propuestas se dirigen a aquellos por tanto a lo que nosotros suponemos de su estado de espíritu. Crapanzano nos había advertido, por consiguiente, con la frase ya citada que evoca «la comprensión 
de nuestros interlocutores» (Crapanzano, 2012: 19). Pero estas innovaciones no hacen más que prolongar las intuiciones de Lisón. Ellas confirman sobre todo la fecundidad de su apuesta, la pertinencia de la vía que ha escogido. Él no pretendió parar de suscitar la creatividad.

Traducción: José Antonio González Alcantud

\section{REFERENCIAS BIBLIOGRÁFICAS}

Althabe, G. (1969). Oppression et libération dans l'imaginaire. París: François Maspero.

Auerbach, E. (2000). Mimesis. La représentation de la réalité dans la littérature occidentale. París: Gallimard.

Campagne, J. (2012). Tu ne m'a pas jetée, c'est moi qui suis partie. Lormont: Le Bord de l'eau, Des mondes ordinaires.

Chauvier, E. (2004). Profession anthropologue. París: William Blake and C\%/Art et Arts.

Clifford, J. (1988). The Predicament of Culture. Twentieth-Century Ethnography, Literature, and Art. Cambridge, Massachusetts; London, England: Harvard University Press.

Crapanzano, V. (2012). Les Harkis. Mémoires sans issue. París: Gallimard.

Duranti, A. (1994). From Grammar to Politics. Berkeley, Los Angeles, London: University of California Press.

Geertz, C. (1973). The Interprétation of culture. London: Fontana Press.

Ginzburg, C. (1980). Le fromage et les vers. L'univers d'un meunier du XVle siècle. París: Flammarion.

Gluckman, M. (1940). Analisis of a social situation in modern zululan. Bantu Studies, 14(1), pp. $1-30$.

Jablonka, I. (2017). L'hitoire est une littérature contemporaine. París: Points.

Jules-Rosette, B. (1996). L'ethnographe et l'ethnologie indigène: l'histoire, le discours et les paysages de la mémoire. Cahiers Ethnologiques, 18.

Le Roy Ladurie, E. (1975). Montaillou, village occitan. París: Gallimard.

Levinson, S. C. (1983). Pragmatics. Cambridge: Cambridge University Press.

Lisón Tolosana, C. (1966). Belmonte de los Caballeros. Oxford: Clarenda.

Lisón Tolosana, C. (1971). Antropología social en España. Madrid: Siglo XXI.

Lisón Tolosana, C. (1974). Perfiles Simbólicos y Morales de la Cultura Gallega. Madrid: Akal.

Lisón Tolosana, C. (1990). Demonios y exorcismos en los siglos de oro. Madrid: Akal.

Lisón Tolosana, C. (1998). La Santa Compaña. Fantasías reales, realidades fantásticas (Antropología cultural de Galicia IV). Madrid: Akal. Traduction française Réalités fantastiques, Pessac: Presses Universitaires de Bordeaux, Etudes culturelles, 2007.

Lisón Tolosana, C. (2005). La fascinación de la diferencia. La adaptación de los Jesuitas al Japón de los samuráis, 1549-1592. Madrid: Akal.

Malinowski, B. (1974). Théorie ethnographique du langage. En: Les jardins de corail. París: François Maspero, Textes à l'appui.

Milhe, C. (2020). Le mystère de la cagoule. Enquête bolivienne, Toulouse: Anacharsis.

Traimond, B. (2004). La polémique Sartre/Lévi-Strauss revisitée en La mise à jour. Introduction à l'ethnopragmatique. Pessac: Presses Universitaires de Bordeaux, Etudes culturelles. 
Traimond, B. (2008). L'anthropologie à l'époque de l'enregistrement de paroles. Bordeaux, William Blake and $\mathrm{C}^{\circ} /$ Art et Arts.

Traimond, B. 2(012). La novedad de la obra de Carmelo Lisón Tolosana, Anthropos, 235.

Traimond, B. (2016). Qu'est-ce que l'ethnopragmatique? Passac: Presses Universitaires de Bordeaux, Etudes culturelles.

Visweswaran, K. (1994). Fictions of Feminist Ethnography. Minneapolis, London: University of Minneapolis Press. 